\title{
A BI-DIRECTIONAL MAGNETIC MICROPUMP ON A SILICON WAFER
}

\author{
Wenjin Zhang and Chong H. Ahn \\ University of Cincinnati \\ Center for Microelectronic Sensors and MEMS \\ Department of Electrical and Computer Engineering and Computer Science \\ P.O. Box 210030, Cincinnati, Ohio 45221-0030
}

\begin{abstract}
A new electromagnetically driven peristaltic micropump on a silicon wafer with a low driving voltage less than 3 volts has been designed, fabricated, and tested. To construct the micropump, electromagnets on a Pyrex glass wafer and micropump parts on a silicon wafer are separately fabricated, and then assembled together using low temperature polymer bonding techniques. This new fabrication technique of magnetic microactuators using low temperature post-bonding techniques provides a flexible and unique approach in realizing various magnetic MEMS devices. The realized magnetic micropumps have been functionally operated in peristaltic motions with a DC $300 \mathrm{~mA}$ and 3 volts allowing bi-direction pumping flows. The maximum attainable flow rate was approximately $20 \mu \mathrm{l} / \mathrm{min}$ at $5 \mathrm{~Hz}$. In this work, a new electromagnetically driven bi-directional micropump has been successfully realized on silicon wafers.
\end{abstract}

\section{INTRODUCTION}

There has been a large demand for a micropump which has a high flow rate with a low driving voltage. Most micropumps which have been realized to date are suffering from high driving voltages to attain high flow rates depending on their application targets. In specific, if a micro fluid regulation system is sharing a power supply with signal control or processing IC chips which require less than 3-5 volts, low driving voltage of micropumps is critically essential in practical applications. If the driving voltages of the micropump require those high values that would not be attainable from the power supply with the IC chips, the micropump system must require an extra power supply or voltage regulator. Furthermore, micro fluid regulation systems for biomedical or biological applications such as portable or implantable drug delivery systems require low driving voltages, since electrical breakdown hazard from the high voltages is seriously concerned due to its safety issues.

Micropumps which have a function of bi-directional fluid driving mechanism are usually required in biomedical or chemical analysis systems [1], where the bi-directional pumping actions are using to produce either a washing flow or a sampling flow. For a preliminary bi-directional magnetic micropump, a peristaltic micropump [2-4] using magnetic variable-reluctance principles has been designed and fabricated in this work. Thus, the micropump fabricated in this work consists of an electromagnetically actuated pump chamber and two peristaltic microvalves as shown in Figure 1 . By controlling the exciting sequence of the two valves and the pump chamber, its pumping operation can be achieved as well as its flow directions can also be changed. Magnetic forces have been used for the microactuators which require low driving voltages as well as large driving forces [5-7].
In this work, a new proto-type magnetic microactuator as shown in Figure 2 has been proposed and realized using both bulk micromachining and wafer bonding techniques, where magnetic forces can be produced between the electromagnets and the permalloy films plated on silicon diaphragms (i.e., permalloy/silicon diaphragm or magnetic diaphragm). When a current is applied to the inductor mounted on a glass wafer, the permalloy/silicon diaphragm is attracted toward the upper electromagnet, lifting the valve bosses and thus opening the valve as described in [8]. In realizing this structure, the planar inductors and the magnetic diaphragms are separately fabricated and then assembled using low temperature polymer bonding techniques.

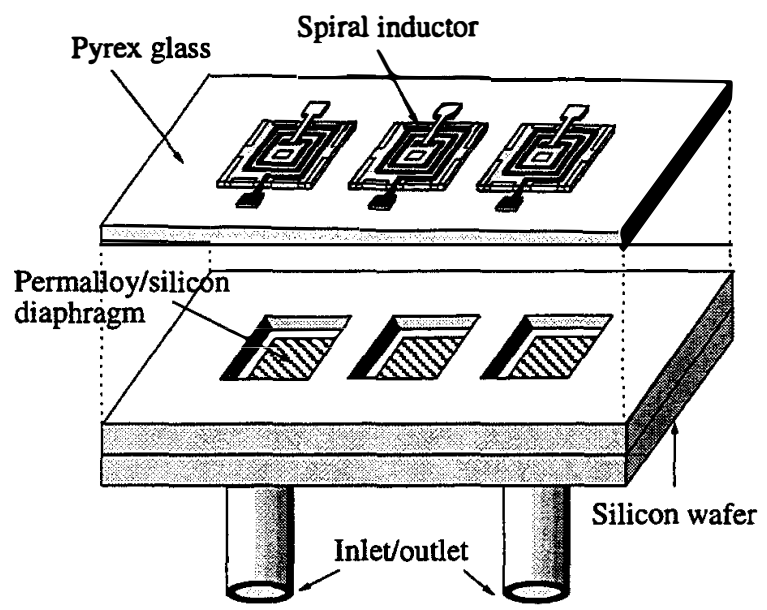

Figure 1. Schematic diagram of a magnetically driven bidirectional micropump.

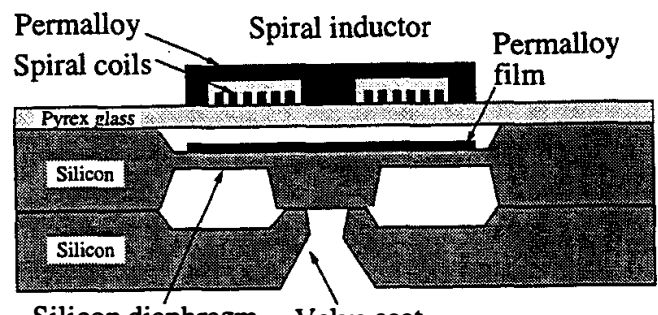

Silicon diaphragm Valve seat

Figure 2. Schematic diagram of a prototype magnetic microactuator providing vertical actuation. 
Pyrex glass wafers are serving as an inductor substrate as well as an insulation substrate which passes magnetic flux through it. The magnetic diaphragms and valve chambers are fabricated using conventional bulk micromachining and low temperature eutectic bonding techniques. Thus, a new fabrication technique in realizing magnetic microactuators based on the combination of bulk micromachining and wafer bonding techniques has been introduced, which provides a flexible and unique approach in the fabrication of magnetic microactuators. In this work, a new magnetically driven bi-directional micropump on a silicon wafer with a low driving voltage of 3 volts has been designed, fabricated, and tested.

\section{INDUCTOR AND PUMP DESIGN}

Several micromachined planar inductors for the applications of microactuators have been reported [9-11]. Among them, the planar spiral-type inductor [9] has a very favorable structural affinity to the structure of micropumps, microvalves, and several planar microactuators which usually require a vertical actuation of diaphragm. The major concerns in designing this type of inductor are to achieve a high inductance, a low coil resistance, and a low magnetic field interference. In the spiral inductor structure, if magnetic cores with a high permeability encapsulate spiral coils constructing a closed magnetic circuit, the achievable inductance value increases due to the low reluctance of the inter-linked magnetic circuit between the coils and the cores. A micromachined planar spiral inductor which has fully encapsulated magnetic cores was reported in [9]. Accordingly, the magnetic field interference can be minimized, confining the magnetic fluxes in the closed magnetic core. However, the bottom magnetic core from the fully encapsulated inductor is simply removed from the inductor proposed in this work for micropump applications. As described in Figure 2, magnetic path will be completed through the magnetic core electroplated on a silicon diaphragm (i.e., permalloy/silicon diaphragm). The magnetic forces generated in this actuator depend on the variation of magnetic reluctance through this magnetic path including air gaps. A designed spiral inductor to be fabricated on a Pyrex glass wafer is described in Figure 3, where spiral conductor lines are encapsulated from the top with an opening path at the bottom.

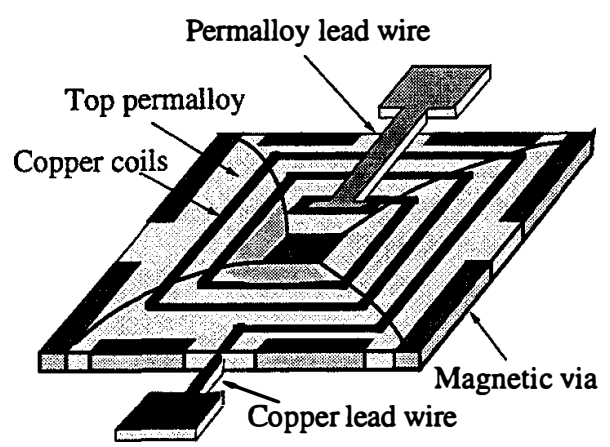

Figure 3. Schematic diagram of the designed spiral inductor.

In order to allow bi-directional fluid flow, this micropump is designed to have a pump actuator and two peristaltic microvalve actuators as shown in Figure 4. The top wafer is used for diaphragms and central bosses, and the bottom wafer is used for valve seats, flow channels, and inlet and outlet holes. The valves consist of a diaphragm with a central boss which mates to an etched silicon valve seat, composing normallyclosed diaphragm valves [8]. Silicon diaphragms have a size of $8 \mathrm{~mm} \times 8 \mathrm{~mm}$. Micro flow channels which have a size of 1000 $\mu \mathrm{m} \times 200 \mu \mathrm{m}$ are designed to connect between the chambers, and the inlet and outlet holes are designed to have a dimension of $500 \mu \mathrm{m} \times 500 \mu \mathrm{m}$.

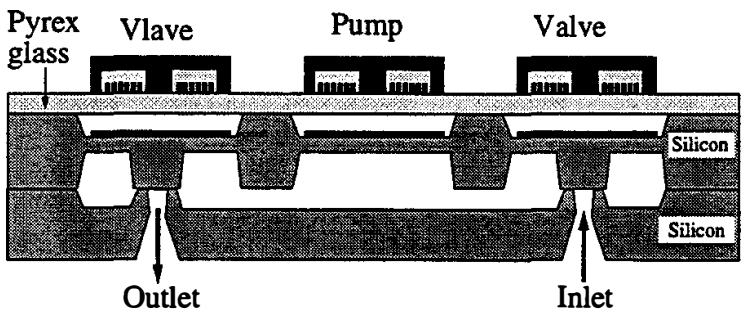

Figure 4. Cut-view of the designed micropump.

\section{FABRICATION}

Planar spiral inductors were fabricated using AZ-4000 series thick-photoresist with three mask process. The brief fabrication steps are described in Figure 5.

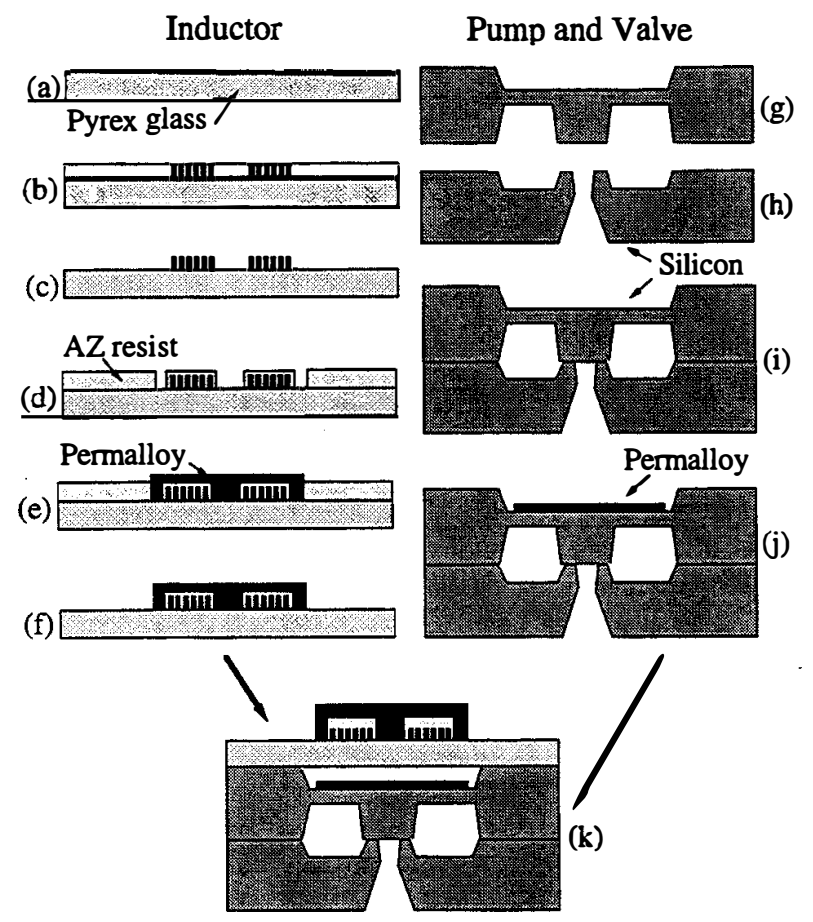

Figure 5. Fabrication steps of inductors and pump parts: (a) deposit seed layer; (b) plate bottom conductor lines; (c) strip PR and seed layer; (d) insulation layer; (e) plate permalloy; (f) strip PR; (g) etch pump chamber; (h) etch valve seat; (i) eutectic bonding; (j) plate permalloy film; (k) assemble two components. 
The fabrication of the inductor started with 2-inch diameter and 10 mil thick Pyrex 7740 glass wafers. Onto the glass wafers, chromium $(300 \AA) /$ copper $(3000 \AA) /$ chromium (200 $\AA$ ) was deposited to form a seed layer for electroplating. AZ-4000 photoresist was used to build the plating molds of spiral conductors with a thickness of $40 \mathrm{um}$, and then copper was electroplated through the molds. Upon completion of the plating, the photoresist was stripped with acetone, and the seed layer was then removed to isolate the spiral coils from each other. AZ-4000 photoresist was spun on the spiral coils, patterned for conductor via holes, and then cured at $150{ }^{\circ} \mathrm{C}$ for 1 hour to make a planarized insulation layer. Onto the cured photoresist, chromium (300 $\AA$ ) / copper $(3000 \AA) /$ chromium $(200 \AA)$ was deposited again for the seed layer of the top magnetic core plating. To electroplate simultaneously both the top magnetic core and the permalloy lead wire from vias to bonding pads, the similar processes used in fabricating the spiral conductors were repeated. Figure 6 shows the photomicrograph of the fabricated spiral inductor.

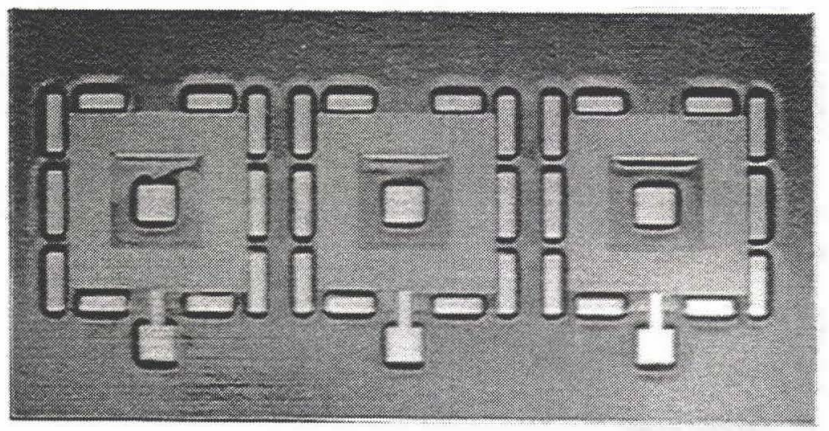

Figure 6. Microphotograph of the fabricated spiral inductor which is taken from rear side through the glass wafer.

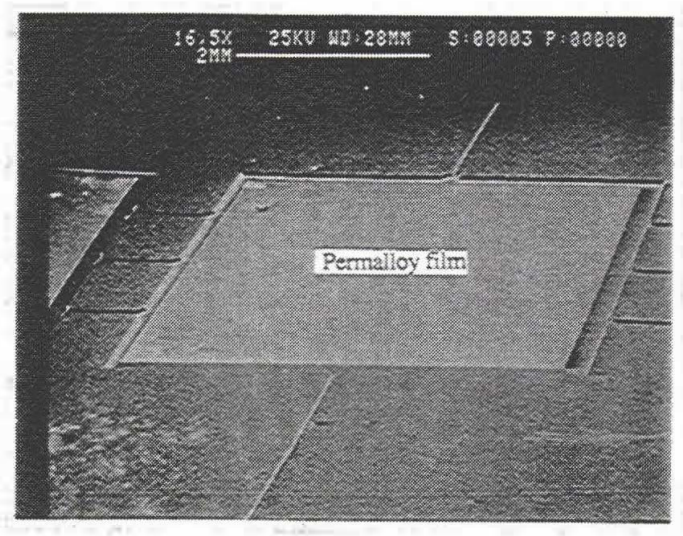

Figure 7. SEM of the fabricated pump part, where the permalloy/silicon diaphragm is shown.

On the other hand, the fabrication of the pump parts started with 2 -inch $\{100\}$ silicon wafers with a silicon oxide of $1.0 \mu \mathrm{m}$ following the steps described in Figure 5. Diaphragms and central bosses were fabricated on the top wafer using bulk micromachining techniques in $\mathrm{KOH}$ solution. To provide an actuation gap between the glass wafer and the silicon diaphragm, a silicon of $30 \mu \mathrm{m}$ was etched from the top of the diaphragm, which allows an ultimate diaphragm thickness of 10 $\mu \mathrm{m}$. Valve seats, flow channels, and inlet and outlet holes were also anisotropically etched on the bottom wafers.

To bond two wafers using gold eutectic bonding techniques, both wafers were deposited with titanium $(300 \AA)$ / gold (3000 $\AA$ ) layer and then bonded together at $380^{\circ} \mathrm{C}$ for 10 minutes. Upon the completion of the bonding, chromium ( $300 \AA) /$ copper $(3000 \AA)$ / chromium (200 $\AA$ ) seed layer was deposited on the top of the diaphragm for the plating of the magnetic film. A nickel-iron permalloy of $7 \mu \mathrm{m}$ thick was plated as a magnetic film on the silicon diaphragm $[6,12]$ to construct a permalloy/silicon diaphragm. Figure 7 shows scanning electron micrograph of the bonded diaphragm and valve seats, where the diaphragm has a size of $8 \mathrm{~mm} \times 8 \mathrm{~mm} \times 17 \mu \mathrm{m}$ in square shape.

Finally, the separately fabricated spiral inductors and pump parts were assembled using low temperature polymer bonding techniques [13]. The micropump were diced into chips and glass pipes were attached on both the inlet and the outlet. Figure 8 shows the photograph of the micropump mounted on a packaging for test.

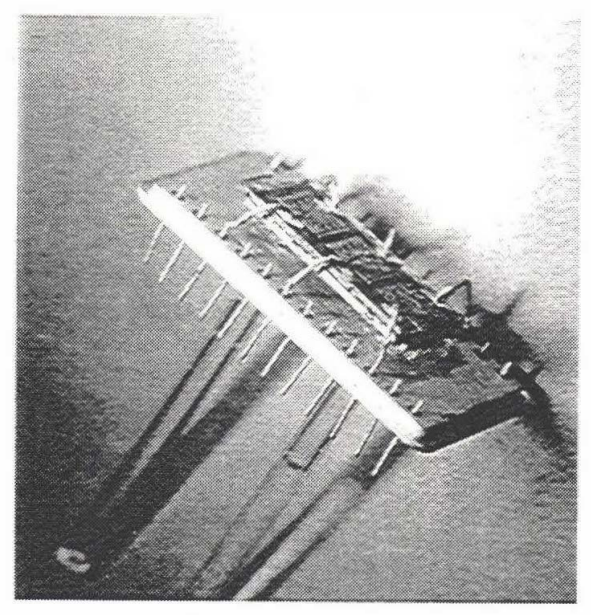

Figure 8. Microphotograph of the assembled micropump which is mounted on the package for test.

\section{EXPERIMENTAL RESULTS}

To evaluate the fabricated inductors, the planar inductors fabricated on the glass wafers were diced into chips. The chip was mounted on a ceramic package, and then the inductance of the device was measured using an HP-4192A impedance analyzer. For a typical 20-tum device of $8 \mathrm{~mm} \times 8 \mathrm{~mm}$ in area, an inductance of approximately $1.7 \mu \mathrm{H}$ was measured at $10 \mathrm{KHz}$ from the fabricated spiral inductor. The measured inductance versus frequencies is plotted in Figure 9. The measured conductor resistance was approximately 3 ohms which allows a high exciting current with even low driving voltage less than 3 volts.

A preliminary pumping test was performed for the fabricated micropump using DI water. The DI water was supplied through the inlet and outlet glass pipes with an inside diameter of $1.5 \mathrm{~mm}$. Using a power MOSFET micropump driving circuit, three phase square waves were applied to the pump in keeping the exciting sequence to achieve a sequential peristaltic micropump action. The pumping flow rate of the pump was evaluated by measuring the total amount of pumped fluid for a given duration at a fixed driving frequency. The measurements 
were repeated over a frequency range of magnetic diaphragm actuation from $1 \mathrm{~Hz}$ to $20 \mathrm{~Hz}$.

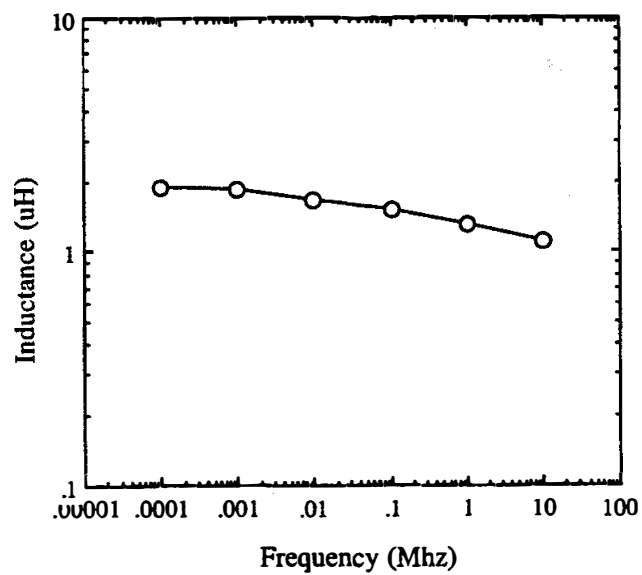

Figure 9. Inductance of the fabricated inductor as function of frequencies.

A maximum flow rate of $20 \mu \mathrm{l} / \mathrm{min}$ was attained at $5 \mathrm{~Hz}$. By changing the phase firing order in reverse, the direction of pumping flow was also changed in reverse achieving the bidirectional micropump function. Flow rate, developed pressure, and power consumption as a function of the driving frequencies and voltages are currently being investigated.

\section{CONCLUSION}

A new magnetic driven bi-directional peristaltic micropump on a silicon wafer with a low driving voltage less than 3 volts has been designed, fabricated, and tested. Using low temperature post-bonding techniques, the fabrication techniques demonstrated in this work provide a flexible and unique approach in realizing various magnetic MEMS components. The realized magnetic micropumps have been functionally operated with a DC $300 \mathrm{~mA}$ and 3 Volts showing bi-direction pumping flows. The maximum attainable flow rate was approximately $20 \mu \mathrm{l} / \mathrm{min}$ at $5 \mathrm{~Hz}$. In this work, a new electromagnetically driven bi-directional micropump has been successfully realized on Pyrex glass and silicon wafers.

\section{ACKNOWLEDGEMENT}

The authors wish to thank Profs. H. Thurman Henderson and Joseph $\mathrm{H}$. Nevin and all members of Center for Microelectronic Sensors and MEMS at the University of Cincinnati for their help and technical discussion. The authors would also like to gratefully acknowledge Hoechst Celanese Co., DuPont, OCG Microelectronics Materials, and DOW Plastics for their donations of materials.

\section{REFERENCES}

[1] V. Gass, B. H. van der Schoot, S. Jeanneret, and N. F. de Rooij, "Integrated Flow-Regulated Silicon Micropump", Sensors and Actuators A, Vol. 43, pp. 335-338, 1994.
[2] J. A. Folta, N. F. Raley, and W. E. Hee, "Design, Fabrication and Testing of a Miniaturized Peristaltic Membrane Pump", Proc. IEEE Solid-State Sensors and Actuators Workshop, pp. 186-189, 1992.

[3] R. Zengerle, S. Kluge, M. Richter, and A. Richter, "A Bidirectional Silicon Micropump", Proc. IEEE Microelectromechanical Systems Workshop, pp. 19-24, 1995.

[4] J. G.Smiths, "Piezoelectric Micropump with Three Valves Working Peristaltically", Sensors and Actuators, A21-A-23, pp. 203-206, 1990.

[5] K. Yanagisawa, H. Kuwano, and A. Tago, "An Electromagnetically Driven Microvalve", Proc. Transducers'93, pp. 102-105, 1993.

[6] C. H. Ahn, Yong. J. Kim, and Mark. G. Allen, "A Planar Variable Reluctance Magnetic Micromotor with Fully Integrated Stators", IEEE/ASME Journal of Microelectromechanical Systems, Vol. 2, No. 4, pp. 165173, 1993.

[7] C. H. Ahn and M. G. Allen, "Fluid Micropumps Based on Rotary Magnetic Actuators", Proc. IEEE Microelectromechanical Systems Workshop, pp. 408-412, 1995.

[8] H. Jerman, "Electrically-Activated, Normally-Closed Diaphragm Valves", Journal of Micromechanics and Microengineering, Vol. 4, No. 4, pp. 210-216, 1994.

[9] C. H. Ahn and M. G. Allen, "A Planar Micromachined Spiral Inductor for Integrated Magnetic Microactuator Applications", Journal of Micromechanics and Microengineering, Vol. 3, pp. 1-9, 1993.

[10] C. H. Ahn and M. G. Allen, " A New Toroidal-Meander Type Integrated Inductor with Multilevel Meander Magnetic Core", IEEE Transactions on Magnetics, Vol. 30, No. 1, pp. 73-79, 1994.

[11] C. H. Ahn, Y. J. Kim, and M. G. Allen, "A Fully Integrated Planar Toroidal Inductor With A Micromachined Nickel-Iron Magnetic Bar", IEEE Transactions on Components, Hybrids, and Manufacturing Technology, Vol. 25, No 3, pp. 356403, 1994.

[12] R. L. Anderson, E. E. Castellani, P. M. McCaffrey, and L. T. Romankiw, "Method for Treating Magnetic Alloy to Increase The Magnetic Permeability", United States Patent $\# 4,003,768$

[13] J. C. Bolger and C. T. Mooney, "Die Attach in Hi-Rel PDips: Polyimides or Low Choride Epoxies", Proc. IEEE Electronics Components, pp. 63-67, 1984. 